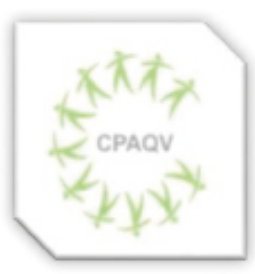

ISSN: 2178-7514

Vol. 12 | No. 3| Ano 2020

ARTIGO ORIGINAL

\title{
PERCEPÇÃO DE PROFISSIONAIS E ACADÊMICOS DE EDUCAÇÃO FÍSICA SOBRE O TREINAMENTO CROSSFIT NA CIDADE DE PORTO VELHO-RO
}

High intensity training for physical conditioning: perception of physical education professionals in the city of Porto Velho - RO

Vanessa Rityele Moura da Silva ${ }^{1}$; Ricardo Pablo Passos²; Luís Felipe Silio ${ }^{1,2,4}$; Érika de Araújo Almeida ${ }^{1}$; Luis Fernando de Oliveira Lima ${ }^{1}$; José Ricardo Lourenço de Oliveira ${ }^{2}$; Heleise Faria dos Reis de Oliveira ${ }^{3}$; Carlos Henrique Prevital Fileniं ; Guanis de Barros Vilela Junior ${ }^{2}$; Elizângela de Souza Bernaldino ${ }^{1,4}$

\section{RESUMO}

A presente pesquisa teve por objetivo conhecer a percepção dos profissionais de Educação Física acerca da modalidade esportiva CrossFit da cidade de Porto Velho - RO. Para tanto foi realizada uma pesquisa de campo do tipo descritiva com 20 profissionais e estudantes de Educação Física que atuam em academias e/ou Box de CrossFit na cidade de Porto Velho - RO. Como instrumentos de coleta de dados utilizou-se um questionário estruturado com 10 (dez) questões mistas. A pesquisa foi submetida ao Comitê de Ética em Pesquisa e mediante sua aprovação a coleta de dados foi iniciada junto aos profissionais de Educação Física e/ou estudantes em horário previamente acordado (antes e/ou após o horário de trabalho). Para análise dos dados recorreu-se a estatística descritiva percentual para análise do objeto "conhecimento sobre os benefícios do treinamento de alta intensidade". Como resultados predominantes: quanto ao conhecimento da modalidade e dos fundamentos: obteve-se $(100 \%)$ de respostas afirmativas no grupo de acadêmicos e profissionais CrossFit (CF); e respectivamente (93,3\%) e $(60 \%)$ no grupo de acadêmicos e profissionais de academias (AC). Referente ao risco de lesões: (100\%) do grupo CF afirmaram ser passivo de lesão como qualquer outra modalidade; e $(60 \%)$ do grupo AC destacaram que tem risco eminente de lesão. Sobre a forma como tomou conhecimento do CF: no grupo CF (40\%) em competições, enquanto que no grupo AC, $(33,3 \%)$ relataram que conheceram a modalidade por meios diversificados. Em relação à afirmação de que a modalidade ocupa o espaço de outra área, ambos os grupos apontaram que não $(60 \%$ no CF e 87,6 no AC). No que diz respeito à percepção dos grupos sobre o treinamento CF: no grupo CF (80\%) revelaram ser um novo campo de atuação; no grupo AC (40\%) afirmaram que tem objetivos diferentes do convencional. Em se tratando dos benefícios e motivos para adesão e permanência ao CF: houve unanimidade acerca dos benefícios do CF para saúde (100\%); (40\%) apontaram que aderiram por conta da eficiência dos resultados; $(40 \%)$ permanecem pelo senso da comunidade no ambiente do CF. Em síntese, os achados a respeito dos profissionais sobre a modalidade CF mostrou que o CF não é mal visto pelos profissionais de musculação, entretanto há necessidade de maiores esclarecimento e discussões referentes aos riscos relacionados a lesões e a metodologia de treinamento $\mathrm{CF}$, atingindo assim o objetivo primário da pesquisa. Palavras-chave: Treinamento; Alta Intensidade; CrossFit; Profissional de Educação Física.

\begin{abstract}
This research aimed to know the perception of Physical Education professionals about the CrossFit sport modality in the city of Porto Velho - RO. For this purpose, a descriptive field research was carried out with 20 professionals and students of Physical Education who work in gyms and / or CrossFit Box in the city of Porto Velho - RO. As instruments of data collection, a structured questionnaire with 10 (ten) mixed questions was used. The research was submitted to the Research Ethics Committee and upon its approval, data collection was initiated with Physical Education professionals and / or students at a previously agreed time (before and / or after working hours). For data analysis, we used the percentage descriptive statistics to analyze the object "knowledge about the benefits of high intensity training". As predominant results: regarding the knowledge of the modality and the fundamentals: it was obtained (100\%) of affirmative answers in the group of academics and professionals CrossFit (CF); and respectively (93.3\%) and (60\%) in the group of academics and academy professionals (AC). Regarding the risk of injury: (100\%) of the CF group stated that they are passive of injury like any other modality; and (60\%) from the AC group highlighted that they have an imminent risk of injury. About the way he learned about FC: in the CF group $(40 \%)$ in competitions, while in the AC group, $(33.3 \%)$ reported that they got to know the sport through different means. Regarding the statement that the modality occupies the space of another area, both groups indicated that it was not ( $60 \%$ in the FC and 87.6 in the AC). Regarding the perception of the groups about the CF training: in the CF group (80\%) they revealed to be a new field of action); in the AC group ( $40 \%$ ) they stated that they have different objectives than the conventional one. In terms of the benefits and reasons for joining and staying at the FC: there was unanimity about the benefits of FC for health $(100 \%) ;(40 \%)$ pointed out that they adhered because of the efficiency of the results; $(40 \%)$ remain in the sense of the community in the FC environment. In summary, the findings regarding the professionals about the FC modality showed that the FC is not frowned upon by the bodybuilding professionals, however there is a need for further clarification and discussions regarding the risks related to injuries and the FC training methodology, thus reaching the primary research objective.
\end{abstract}

Keywords: Training; High Intensity; CrossFit; Physical Education Professional.

\author{
1. Centro Universitário São Lucas \\ 2. Universidade Metodista de Piracicaba (UNIMEP) \\ 3. Universidade Estadual de Ponta Grossa (UEPG) \\ 4. Universidade federal de Rondônia (UNIR)
}

Autor de correspondência

Elizângela de Souza Bernaldino

E-mail:es-bernaldino@hotmail.com 


\section{INTRODUÇÃO}

O profissional de Educação Física tem que estar atento às tendências dentro do campo de atuação, visto que o mercado de trabalho está cada vez mais competitivo e amplo. Alguns programas de treinamentos em alta intensidade ou condicionamento extremo vêm de uma crescente nos últimos tempos, ganhando assim a atenção do público (1).

Os programas de condicionamento extremo são caracterizados por movimentos funcionais de alta intensidade e constantemente variados. Os treinamentos tem como base o estímulo de superação e auto desafio levando o praticante à exaustão durante as sessões do treino, logo, os programas são compostos por fatores motivacionais e competitivos. Além disso, o fato do treinamento ser versátil e eficaz, a modalidade vem conquistando novos adeptos em busca da sua melhor versão, principalmente os que estão saturados das atividades monótonas (1).

Como exemplos de treinamentos para o condicionamento extremo, pode-se citar o HIIT e o CF que tem por finalidade desenvolver aptidão física cardiovascular, força e força explosiva. Como ponto de destaque, nestes tipos de treinamento, o praticante tem que executar na maioria das vezes exercícios combinados, com um número de repetições pré-estabelecidas no menor tempo possível ou no tempo previsto, o tempo de recuperação é curto ou inexistente entre as séries (1).
O treinamento de CF envolve movimentos funcionais em alta intensidade combinados com exercícios de força, aptidão cardiorrespiratória, força explosiva e movimentos ginásticos, tais como: levantamento terra, agachamento, desenvolvimento, LPO, flexões, abdominais, burppes, bike, nado, remos, corridas, arremesso, saltos, pull-ups, e subida na corda.

Quanto aos resultados e/ou efeitos do treinamento para o condicionamento extremo, Prestes et al (2) realizaram um comparativo entre uma sessão de treinamento convencional e uma sessão de CrossFit (CF) e identificaram como principais diferenças a estruturação do programa de treino. Dentre as mudanças, percebeu-se que no treinamento convencional, os programas são alternados por segmentos, articulações e por grupamento muscular, de forma seriada, onde a execução de cada série respeita seu intervalo, com ênfase no respectivo grupo muscular que é dividido por sessões de treinamento. Os exercícios são realizados em aparelhos, barras e pesos livres. Enquanto que no treinamento CF são obedecidas três fases: aquecimento, técnica e WOD.

Sobre as fases do treinamento CrossFit, Tibana e Prestes (1) explica que o WOD "workout of the day" chamado de treinamento do dia, é composto por uma série de sequência pré-estabelecida. Inicialmente é realizado o treino trabalhando mobilidade articular seguido de aquecimento e preparação 
ao movimento, um exercício para desenvolver força ou outra técnica de um movimento específico. Depois dessa sequência é realizado o treino metabólico WOD com exercícios combinados para trabalhar o condicionamento dos praticantes(1).

Com relação ao treino metabólico, o treinamento de CF tem como objetivo desenvolver ao máximo as três vias metabólicas, fosfagênica, glicolítica e oxidativa. Sendo que para trabalhar o condicionamento físico dos praticantes é necessário considerar as 10 habilidades físicas gerais, são elas: resistência cardiorrespiratória, resistência muscular, força, flexibilidade, potência, velocidade, coordenação, agilidade, equilíbrio e precisão. $\mathrm{O}$ seu condicionamento está ligado às habilidades, que serão obtidas através da pratica e do treinamento especifico (3).

A rotina de treinamento em grupo geram grandes benefícios nos adeptos da modalidade, além do treinamento eficaz, é inclusiva, e o praticante vive em constante evolução, superação, buscando-se sempre sua melhor versão tornando o ambiente cada vez mais competitivo e motivador atraindo novos adeptos a modalidade. O treinamento é realizado para que o praticante possa realizar qualquer atividade física. $\mathrm{O} C \mathrm{CF}$ pode ser praticado por atletas até grupos especiais, respeitando a individualidade biológica tendo em vista os efeitos positivo da modalidade tais como, condicionamento e qualidade de vida
(3).

Neste contexto considerando as diferença de uma sessão de treinamento CF para uma sessão convencional, bem como a pouca experiência, a falta de formação adequada e/ou até mesmo o desconhecimento de profissionais referente à modalidade CF, levantou - se o seguinte problema de pesquisa: Qual o conhecimento dos profissionais de Educação Física acerca dos benefícios do treinamento de alta intensidade para o condicionamento físico em praticante de CrossFit na cidade de Porto Velho - RO?.

Sendo assim a presente pesquisa teve por objetivo conhecer a percepção dos profissionais de Educação Física acerca da modalidade esportiva CrossFit da cidade de Porto Velho - RO. Como objetivos secundários, analisar o nível de conhecimento dos profissionais de Educação Física em relação ao treinamento de alta intensidade para o condicionamento físico; verificar se existe preconceito com relação aos novos métodos de treino para o condicionamento que envolve a modalidade CF na cidade de Porto Velho - RO; averiguar a percepção dos profissionais de Educação Física quanto aos benefícios e riscos associados à ocorrência de lesões durante o treinamento na modalidade CF; identificar os motivos para adesão e permanência no treinamento de alta intensidade para o condicionamento na modalidade CrossFit na cidade de Porto Velho $-\mathrm{RO}$.

A realização da pesquisa justifica- 
se pela escassez de estudos na área de CF e pelo crescimento e expansão da modalidade na atualidade como campo de atuação do profissional de Educação Física. Concomitante, percebe-se a necessidade de debate e discussão na literatura referente aos efeitos e benefícios do treinamento de condicionamento extremo. Principalmente no que diz respeito às várias valências e métodos de treinamento que contribuem para que o CF tenha se tornado um diferencial, para a otimização do desempenho e superação dos seus limites de forma prazerosa apesar da alta intensidade, o que se diferencia de outras práticas físicas. (4).

\section{MATERIAL E MÉTODO}

A presente pesquisa trata-se de um estudo do tipo descritiva e quantitativa para fins de levantamento de dados referente à percepção dos professores acerca do objeto "o conhecimento sobre os benefícios do treinamento de alta intensidade" para o condicionamento físico na modalidade CrossFit na cidade de Porto Velho - RO.

Participaram da pesquisa 20 (vinte) profissionais e estudantes de Educação Física de ambos os sexos com idade entre 18 e 40 anos, sendo 11 (onze) profissionais que atuam em academias com musculação; 04 (quatro) estudantes que atuam na musculação; 03 (três) profissionais que atuam em Box no CrossFit e 02 (dois) estudantes que atuam no Cross Fit.

Como instrumentos de coletas de dados, utilizou-se um questionário estruturado pela pesquisadora com 10 (dez) questões mistas. A pesquisa foi submetida ao Comitê de Ética em Pesquisa do Centro Universitário São Lucas Faculdade e obteve aprovação através da CAAE $\mathrm{n}^{\circ}$ 23182719.9.0000.0013. Mediante a aprovação da pesquisa, iniciou-se a aplicação do questionário para coleta de junto aos profissionais e estudantes de Educação Física. Como procedimentos de coleta, houve um contato presencial nas academias e Box com os responsáveis técnicos e coordenadores para fins de explicar os termos e cuidados éticos de acordo com o Termo de Consentimento Livre Esclarecido TCLE. A aplicação do questionário ocorreu durante o intervalo, antes ou depois da jornada de trabalho, de trabalho do profissional e/ou do estudante de Educação Física, posteriormente devido a pandemia, ocorreu a aplicação online dos questionários pelo Google Forms.

Com relação aos critérios de inclusão e exclusão. Foram incluídos na pesquisa Profissionais e acadêmicos/estagiários de Educação Física ativos de ambos os sexos, que atuam na área do treinamento resistido e como Coach (termo usado no CrossFit para treinador/professor), que atuam na cidade de Porto Velho - RO. Com idade entre 18 a 40 anos, que concordarem com a participação na pesquisa mediante a assinatura do TCLE. Foram excluídos da pesquisa: Profissionais de Educação Física e acadêmicos/ estagiário não 
atuante na área, menores de 18 e maiores que 40; Profissionais de Educação Física e estagiários de ambos os sexos de todas a academias e box selecionados na cidade de Porto Velho, que se recusarem a participar da pesquisa e/ou não assinar o TCLE.

Para análise de dados, utilizou-se a estatística descritiva percentual e os resultados foram divididos em dois grupos: Grupo dos estudantes e profissionais do CrossFit e o Grupo dos estudantes e profissionais de musculação. Os resultados foram apresentados em três tabelas.

\section{RESULTADOS}

Para caracterização da amostra, participaram como sujeitos da pesquisa 14 (catorze) profissionais de Educação física de ambos os sexos na faixa etária entre 18 e 40 anos que atuam na profissão por um tempo compreendido entre 01 e 20 anos. Na condição de estudante, participaram 06 (seis) acadêmicos, sendo que 02 (dois) desenvolviam o estágio na modalidade CF e 04 (quatro) em academias de musculação. Com relação ao quantitativo geral, dos 20 profissionais e acadêmicos de Educação Física, $15(75 \%)$ pertencia ao gênero masculino e $05(25 \%)$ ao gênero feminino.

Tabela 1. Percepção dos acadêmicos e profíssionais de Educação Física sobre a modalidade de treinamento CrossFit.

\begin{tabular}{|c|c|c|c|c|}
\hline \multirow[b]{2}{*}{ Variável } & \multicolumn{2}{|c|}{$\begin{array}{c}\text { Estudantes e Profissionais } \\
\text { CrossFit }(\mathbf{n}=\mathbf{5})\end{array}$} & \multicolumn{2}{|c|}{$\begin{array}{c}\text { Estudantes e Profissional } \\
\text { Academias }(\mathbf{n}=\mathbf{1 5})\end{array}$} \\
\hline & $\%$ & $\mathrm{~N}$ & $\%$ & $\mathrm{~N}$ \\
\hline \multicolumn{5}{|l|}{ Conhece a modalidade CrossFit } \\
\hline Sim. & 100 & 5 & 93,3 & 14 \\
\hline Já ouviu falar. & 0,0 & 0,0 & 6,7 & 1 \\
\hline \multicolumn{5}{|c|}{ Conhece as modalidades envolvidas no CrossFit } \\
\hline Sim. & 100 & 5 & 60 & 9 \\
\hline Não. & 0,0 & 0,0 & 6,7 & 1 \\
\hline Já ouviu falar. & 0,0 & 0,0 & 33,3 & 5 \\
\hline \multicolumn{5}{|l|}{ Percepcão sobre risco de lesões } \\
\hline Passivo de lesões específicas. & 0,0 & 0,0 & 6,7 & 1 \\
\hline $\begin{array}{l}\text { Passivo de lesões assim como outras } \\
\text { modalidades. }\end{array}$ & 100 & 5 & 26,7 & 4 \\
\hline Risco iminente de lesões & 0,0 & 0,0 & 53,3 & 8 \\
\hline $\begin{array}{l}\text { Passivo de lesões especificas, assim } \\
\text { como outras modalidades. }\end{array}$ & 0,0 & 0,0 & 6,7 & 1 \\
\hline $\begin{array}{l}\text { Passivo de lesões específicas, risco } \\
\text { iminente de lesões. }\end{array}$ & 0,0 & 0,0 & 6,7 & 1 \\
\hline
\end{tabular}

A tabela1 apresenta a percepção dos profissionais e acadêmicos de Educação Física com relação ao treinamento de alta intensidade na modalidade CrossFit (CF) com base nas variáveis: conhecimento das modalidade CF; Conhece as modalidades envolvidas no CF; Percepção sobre os riscos de lesões. Como resultados, na variável conhecimento da modalidade CF, (93,3\%) dos participantes que trabalham em academias conhecem a modalidade CF e apenas $(6,7 \%)$ já ouviu falar. Sendo que apenas 05 (cinco) participantes indicariam o CF e 06 (seis) não indicaram. Dos participantes que trabalham com o CF no grupo de profissionais e acadêmicos 
de Educação Física que atuam com CrossFit, $(100 \%)$ conhecem a modalidade e indicam, sobre as modalidades foram citados LPO, ginastica, Sprint, natação, condicionamento metabólico e movimentos cíclicos.

Com relação ao conhecimento das modalidades envolvidas no $\mathrm{CF}$, no grupo de profissionais e estudantes que atuam em academias $(60 \%)$ relataram conhecer e $(6,7 \%)$ afirmaram que não conhecem. Dentre as modalidades que constituem o CF, cabe destacar: LPO, ginastica, Sprint e natação. Referente à percepção sobre o risco de lesão, no grupo dos profissionais e estudantes que atuam na musculação como percentuais mais significativos, constatou-se que $(53,3 \%)$ destacaram que há risco eminente de lesões e (26,7\%) afirmaram ser passivo de lesões assim como outras modalidades. e estudantes que atuam com CrossFit (100\%) relataram ser passivo de lesões assim como outras modalidades de treinamento.

Sobre o percentual de profissionais e acadêmicos que afirmaram que a modalidade CF é passível de lesão como qualquer outra modalidade, chama-se atenção acerca das diferenças entre os dois grupos onde (100\%) dos profissionais de CF disseram que o CF é passivo de lesões assim como outras modalidades, e $(53,3 \%)$ apontaram o risco iminente de lesões. A partir dos achados, pode-se inferir que o desconhecimento sobre os métodos de periodização e estruturação do treinamento no CF pode ser um fator influenciador para que um percentual significativo de profissionais $(53,3 \%)$ que atuam na musculação tenham uma percepção negativa que associa o CF como sendo mais passivo a lesão do que as demais modalidades. Em contrapartida, no grupo de profissionais

Tabela 2. Percepção sobre a abrangência e crescimento de treinamento de alta Intensidade na modalidade CrossFit.

\begin{tabular}{|c|c|c|c|c|}
\hline \multirow[b]{2}{*}{ Variável } & \multicolumn{2}{|c|}{$\begin{array}{c}\text { Acadêmicos e Profissionais } \\
\text { CrossFit }(n=5)\end{array}$} & \multicolumn{2}{|c|}{$\begin{array}{c}\text { Acadêmicos e Profissionais } \\
\text { Academias }(\mathbf{n}=15)\end{array}$} \\
\hline & $\%$ & $\mathrm{~N}$ & $\%$ & $\mathrm{~N}$ \\
\hline \multicolumn{5}{|c|}{ Como conheceu a modalidade CrossFit } \\
\hline Por meios diversificados & 0,0 & 0 & 33,3 & 5 \\
\hline Amigos & 20 & 1 & 13,3 & 2 \\
\hline Faculdade & 0,0 & 0 & 20,0 & 3 \\
\hline Internet & 20 & 1 & 13,3 & 2 \\
\hline Competições na cidade & 40 & 2 & 6,7 & 1 \\
\hline Cursos e Especializações & 20 & 1 & 0,0 & 0 \\
\hline Amigos e internet & 0,0 & 0 & 13,3 & 2 \\
\hline \multicolumn{5}{|c|}{ O CrossFit toma espaço de outras áreas } \\
\hline Sim & 40 & 2 & 13,3 & 2 \\
\hline Não & 60 & 3 & 86,7 & 13 \\
\hline \multicolumn{5}{|c|}{ Percepcão sobre o método de treino CrossFit } \\
\hline Novo campo de atuação profissional. & 80 & 4 & 20 & 3 \\
\hline $\begin{array}{l}\text { Possui objetivos diferentes } \\
\text { convencional. }\end{array}$ & 0,0 & 0 & 40 & 6 \\
\hline $\begin{array}{l}\text { Não tenho afinidade com o métoc } \\
\text { treino. }\end{array}$ & 0,0 & 0 & 20 & 3 \\
\hline $\begin{array}{l}\text { Percepção ampliada sobre o métoc } \\
\text { treino. }\end{array}$ & 20 & 1 & 20 & 3 \\
\hline \multicolumn{5}{|l|}{ Eficiência do treinamento CrossFit } \\
\hline Sim & 100 & 5 & 86,7 & 13 \\
\hline Não & 0,0 & 0 & 13,3 & 2 \\
\hline
\end{tabular}


A tabela2 apresenta a percepção dos acadêmicos e profissionais de Educação Física referente à abrangência e o crescimento do treinamento de alta intensidade na modalidade CrossFit (CF). Para tanto se analisou as variáveis: forma como tomou conhecimento da modalidade CF; a percepção sobre o CF ter ocupado o espaço de outra área; a percepção sobre o método de treino CF; e a eficiência do treinamento CF.

Em relação à forma como tomou conhecimento da modalidade $\mathrm{CF}$, no grupo dos acadêmicos e profissionais que atuam em academias, $(33,3 \%)$ conheceram a modalidade CF por meios diversificados; (13,3\%) por amigos; $(20 \%)$ faculdade; (13,3\%) internet; $(6,7 \%)$ competições na cidade; $(13,3 \%)$ conheceram por amigos e faculdade.

No grupo dos acadêmicos e profissionais que atuam com $\mathrm{CF},(40 \%)$ relataram que tomou conhecimento em competições na cidade; (20\%) afirmaram ter conhecido a modalidade através de amigos; (20\%) conheceu na faculdade; e 20\% cursos e especializações.

Referente à percepção de que o CF toma espaço de outras áreas, no grupo de acadêmicos e profissionais de Educação Física que atuam em academias, (13,3\%) responderam que sim; e (86,7\%) afirmaram que não. Quanto à afirmação de que o CF não toma o espaço de outras áreas, os profissionais justificaram que o $\mathrm{CF}$ uniu várias modalidades, deste modo, tornou-se mais uma opção para prática do exercício físico que tem crescido de forma geral, assim como também a expansão do público tem se mostrado relevante dada à vasta metodologia de treino proposta, entretanto, os exercício de musculação também se mantem como base para bons resultados.

No grupo dos acadêmicos e profissionais do $\mathrm{CF},(40 \%)$ evidenciaram que a modalidade vem tomando o espaço de outras áreas do treinamento; $(60 \%)$ relataram que a expansão da modalidade não toma espaço de outras áreas e/ou campos da Educação Física. Com relação aos $40 \%$ que apontaram que o $\mathrm{CF}$ toma espaço de outras áreas, apontaram que por ser uma modalidade de ampla abrangência, que envolve de forma concomitante todas as valências físicas desenvolvendo as aptidões de forma global.

Em contrapartida, os $(60 \%)$ que apontaram que o CF não toma espaço de outras áreas, apontaram que o CF não está tomando espaço de outra área e sim conquistando seu próprio espaço, como ferramenta poderosa no quis diz respeito ao condicionamento e conquistando novos públicos através da diversidade da prática.

Quanto à percepção sobre o método de treino CF, acadêmicos e profissionais de Educação Física que atuam em academias, (20\%) afirmaram ser um novo campo de atuação profissional; $(40 \%)$ relataram que possui objetivos diferentes do convencional; (20\%) destacaram não ter afinidade com o método de treino; e (20\%) demonstraram uma percepção ampliada sobre o método de treino.

Sobre a eficiência do treinamento CF para a performance e condicionamento físico, no grupo dos acadêmicos e profissionais que atuam em academias, (86,7\%) reconheceram a eficiência do CF e (13,3\%) relataram que não ter eficiência. Em relação aos (13,3\%) que apontou a ineficiência do CF, um dos participantes justificou 
a opção pelo fato de o treinamento realizado na academia é melhor e mais efetivo por trabalhar grupos musculares de forma separadas.

Por outro lado, os profissionais que reconheceram a eficiência do treinamento (86,7\%), apontaram que o CF é um esporte de alta intensidade e por se trabalhar todas as valências físicas acabam por contribuir com a melhoria do condicionamento físico dos indivíduos.

$\mathrm{O}$ fato de o treino ser realizado de forma dinâmica e coletiva e apresentar muitas repetições influencia positivamente também na motivação do indivíduo quanto a alcançar bons resultados. Entretanto, um programa de treino bem organizado e periodizado com base no objetivo do indivíduo, independente de ser realizado em academias e/ou em Box de CrossFit atenderá as necessidades do indivíduo.
Com relação à eficiência do treinamento CF no grupo de acadêmicos e profissionais que atuam com o CF, (100\%) dos participantes reconheceram a maior eficácia do treinamento $\mathrm{CF}$, tendo em vista que a partir da programação dos treinos, os ganhos de condicionamento são alcançados e percebidos em todas as capacidades físicas que são desenvolvidas de forma global, sendo, portanto o diferencial do treinamento CF. Outro ponto relevante, diz respeito que a realização de movimentos funcionais constantemente variados e em alta intensidade acaba por provocar maiores estímulos e adaptações fisiológicas ao organismo e ao músculo de forma integrada, o que diferente de outras modalidades onde geralmente se trabalha grupos musculares e valências físicas isoladas.

Tabela 3. Percepção referente aos motivos para adesão e permanência no treinamento CrossFit.

\begin{tabular}{l|c|c}
\multicolumn{1}{c}{ Variável } & \multicolumn{2}{c}{$\begin{array}{c}\text { Acadêmicos e Profissionais } \\
\text { CrossFit (n=5) }\end{array}$} \\
\cline { 2 - 3 } & $\%$ & $\mathrm{~N}$ \\
\hline Motivos para adesão ao CrossFit & 40 & 2 \\
\hline Eficiência nos resultados alcançados. & 20 & 1 \\
\hline Amigos, Competições, Maior eficiência. & 20 & 1 \\
\hline Eficiência nos resultados alcançados e dinheiro. & 20 & 1 \\
\hline Satisfação pessoal. & 100 & 5 \\
\hline A prática do CrossFit trouxe benefícios para saúde & & \\
\hline Sim & 20 & 1 \\
\hline Motivos para permanência na comunidade CrossFit & 40 & 2 \\
\hline Satisfação com a evolução no treino. & 20 & 1 \\
\hline Senso de comunidade no ambiente CrossFit & 20 & 1 \\
\hline Beneficios fisiológicos. & \multicolumn{1}{l}{} \\
\hline Resultado profissional social e financeiro. & & \\
\hline Fonte: Dados da pesquisa, 2020. &
\end{tabular}

A tabela 3 apresenta a percepção referente os motivos para adesão e permanência no treinamento CrossFit com base nas variáveis: os motivos para adesão ao CF; a prática para adesão ao CF; motivos para permanência na comunidade CF. Quanto aos motivos para adesão ao CF. a pratica do CF, (40\%) optaram em virtude da eficiência nos resultados; (20\%) pela influência de amigos, competições e maior eficiência nos resultados; $(20 \%)$ atua na modalidade pela eficiência nos resultados alcançados e pelo dinheiro; $(20 \%)$ pela satisfação pessoal. 
Sobre os benefícios da prática do $\mathrm{CF}$, os profissionais foram unânimes (100\%) quanto ao reconhecimento dos efeitos positivos do CF para a saúde e qualidade de vida das pessoas. Mediante sobre os benefícios do CF para saúde, é possível estabelecer associações positivas das valências físicas, como flexibilidade, força agilidade, resistência cardiorrespiratória, velocidade com o combate do stress, aumento da autoestima, aumento de massa muscular, interação e convívio social, aliados aos benefícios doenças que atacam o psicológico e o emocional como a depressão por exemplo.

Em relação aos motivos para permanência na comunidade $\mathrm{CF}$, como resultados, os acadêmicos e profissionais de Educação Física que atuam com CF apontaram que (40\%) buscam e permanecem por motivos relacionados à interação e o senso de comunidade no ambiente de CF; (20\%) apontaram a satisfação com a evolução do treino; $(20 \%)$ relataram os benefícios fisiológicos alcançados; e (20\%) evidenciaram os resultados percebidos nas esferas profissional, social e financeira.

Com base nos achados, constata-se que o senso de comunidade obteve a maior porcentagem em relação às demais variáveis, bem como o ambiente e a interação social é vislumbrada como ponto de destaque, uma vez que o treino de CF é realizado em grupo o que possibilita trabalhar também as variáveis psicológicas e emocionais do treinamento, e principalmente trabalhar a motivação extrínseca com uma grande aliada para que o indivíduo supere seus limites e alcance uma constante evolução e/ou resultados satisfatórios.

\section{DISCUSSÃO}

Os resultados encontrados na pesquisa referente ao conhecimento dos acadêmicos e profissionais de Educação Física sobre o treinamento de alta intensidade (tabela 1) evidenciou que a grande maioria dos profissionais demostrou conhecimento da modalidade CrossFit (CF) em ambos os grupos, nos profissionais e estudante que atuam $\mathrm{CF}$ e profissionais estudantes que atuam na musculação com base na porcentagem de $(100 \%)$ para um e $(93,3 \%)$ para outro.

Em relação ao conhecimento dos profissionais e acadêmicos sobre a modalidade $\mathrm{CF}$, os achados apontaram que os acadêmicos e profissionais que atuam em academias conhecem apenas parte da modalidade. Tal situação é correlacionada quando se analisa que a percepção dos acadêmicos e dos profissionais que atuam em academias referentes ao risco de lesão (53,3\% optaram por risco iminente de lesão), ou seja, afirmaram que pode ocorrer com maior frequência lesões no CF do que em outras modalidades em virtude de conhecer apenas parcialmente a modalidade.

Segundo Dalfré (5) os riscos de lesões no CrossFit são comparáveis ao de outros esportes, como: Halterofilismo, Atletismo (corridas de longa distância), Rugby, Futebol, Hóquei no gelo e Ginástica. Referente aos resultados acerca da percepção dos acadêmicos e profissionais sobre a abrangência e crescimento de treinamento de alta intensidade CF (tabela 2) observou-se que (40\%) dos profissionais de CF conheceram a modalidade através de competições na cidade, sendo que $(33,3 \%)$ dos profissionais de musculação 
conheceram através de meios diversificados, ou seja, mais de uma variável.

Com relação ao CF tomar espaço de outras áreas, observou-se que $(60 \%)$ do profissional de CF optou por não, sendo que $(86,6 \%)$ dos profissionais da musculação também optaram que não. Neste quesito ambos apontaram que o CF não toma espaço de outras áreas mesmo sendo nova no mercado.

$\mathrm{Na}$ variável percepção sobre o método de treino CF constatou-se (80\%) que apontaram como sendo um novo campo de atuação profissional, sendo que para o profissional da musculação (40\%) apontaram que possui objetivos diferentes do convencional. Corroborando com Ganancio, Cabral e Maoski (6), a ampla variedade da modalidade CF torna esse mercado atrativo aos olhos do consumidor que sempre está em busca de novidades. A maior procura pela modalidade ocorre pelo seu modelo de treinamento e também pela existência de competições; logo, esse variado conjunto de opções que o CF proporciona o torna muito atraente aos olhos do consumidor que não se adapta à monotonia do fitness convencional. Ademais, a eficiência do treinamento CF percebida por ambos os grupos de acadêmicos e profissionais de Educação Física reforçam a eficácia do treino por trabalhar todas as valências físicas, por ser dinâmico e envolver o treino coletivo. Entretanto, independente da musculação e/ou do CF, a estruturação e periodização que envolva o controle adequado dos componentes de treinamento, considerando estímulos fisiológicos e a necessidade do indivíduo, podem gerar respostas e adaptações fisiológicas satisfatórias.

\section{CONCLUSÃO}

A pesquisa possibilitou conhecer a percepção dos profissionais de Educação Física acerca da modalidade esportiva CF na cidade de Porto Velho - RO. Os achados permitiu inferir que os profissionais atuantes com musculação conhecem parcialmente os fundamentos e a modalidade do CF. Tendo em vista que no grupo de profissionais de musculação houve associações significativas entre a percepção sobre a modalidade e o risco de lesão ser maior e/ou mais propenso no CF do que nas demais modalidades.

Os profissionais que trabalham com CF demonstram uma percepção abrangente e fundamentada nos métodos e fundamentos do treinamento CF e reconheceram a eficácia do CF para o desenvolvimento das valências físicas e dos aspectos psicológicos e emocionais. Tal associação é percebida pelo fato que como principal motivo para permanência na modalidade é o fator motivacional desencadeado pelo trabalho coletivo, a superação de limites e o senso de comunidade no ambiente.

Os achados permitiram também inferir que o CF não é de fato mal visto pelos profissionais de musculação, entretanto há necessidade de maiores esclarecimento e discussões referentes aos riscos relacionados a lesões e a metodologia de treinamento CF. Percebeu-se também a preferência e/ou inclinação dos profissionais quanto a atuar em academias, o que acabou por impor limitações ao profissional que por motivos diversificados não busca e/ou tem interesse em conhecer com profundidade os métodos de treino da modalidade CF. 
Em síntese, recomenda-se a realização de novos estudos com uma amostra maior de profissionais do $\mathrm{CF}$, bem como a realização de novas pesquisas para fins de aprofundar a análise sobre a percepção dos profissionais de Educação Física e acadêmicos referente ao CF.

\section{REFERÊNCIAS}

1.Tibana RA, Almeida LA and Prestes J. Crossfit riscos ou benefícios, o que sabemos até o momento? Rev Bras Ciencia Mov. 2015; 23: 182-5.

2.Prestes J, Foschini D, Marchetti PH, Charro MA. (2010) Prescrição e periodização do treinamento de força em academias. São Paulo: Manole.

3.Glassman, G. O Guia de treinamento CrossFit. CrossFit Journal, [on-line], 13 jul. 2014. Disponível em: <https://goo.gl/7B1wew>. Acesso em: 08 abr. 2019.

4.Guia de treinamento de nível 1 crossfit. Disponível em: $<$ http://library.crossfit.com/free/pdf/CFJ_L1_TG_ Portuguese.pdf $>$. Acesso em: 25 de agosto de 2019.

5.Dalfré, O. (2017) crossfit e as incidências de lesões: análise crítica. 2017. No39. Trabalho de Conclusão de Curso (Graduação em Ciências do Esporte.) Faculdade de Ciências Aplicadas. Universidade Estadual de Campinas. Limeira.

6.Ganancio MA, Cabral AP, Maoski B. (2018). Análise da Gestão de Negócios no Crossfit em Curitiba/PR Rev de Gestão e Negócios do Esporte (RGNE) - ISSN 2448-3052 Vol. 3 - N. 2, 173-188..

OBSERVAÇÃO: Os autores declaram não existir conflitos de interesse de qualquer natureza. 